\title{
Synchronization of regional public transport systems
}

\author{
I. Schuele, M. Schroeder \& K. H. Kuefer \\ Fraunhofer Institute for Industrial Mathematics, \\ Kaiserslautern, Germany
}

\begin{abstract}
Transferring from one vehicle to another is generally inevitable in public transportation. In urban areas this often includes changing between vehicles operated by different companies. It is well known that good transfer possibilities increase the attractiveness of public transportation. Since this is an important goal, in the present paper we study the synchronization of timetables between different operators with an individual timetable planning process. The synchronization task is a very complicated problem due to the large amount of transfer locations and possibilities and the fact that they are interrelated network-wide. Our main contributions are a model to evaluate transfer quality locally and globally and an interactive optimization approach that allows an integration of the power of mathematical optimization algorithms with the expertise of the planners. We demonstrate our approach by highlighting some results achieved for the public transportation network of the city of Kaiserslautern, situated in south-western Germany.
\end{abstract}

Keywords: public transport synchronization, optimization, decision support.

\section{Introduction}

The need for synchronizing timetables occurs when several public transport companies interact within the same regional area. Generally, the companies offer good transfer possibilities to their passengers inside their own networks, but there is often a lack of synchronization at the intersection points of the different companies.

In our concept for optimizing public transport timetables, the goal is to synchronize a set of transportation lines by changing their starting times to 
ensure that passengers can transfer more conveniently. For that reason, we replace the often used approach of simply minimizing waiting times by a concept that tries to achieve transfers that can be called convenient. For these transfers, the waiting times should not be too long, but also not too short, in order to reduce the risk of missing a transfer if the arriving vehicle is delayed.

The large number of transfer possibilities between the different companies and the interdependencies within the network are practically impossible to handle by a manual planning procedure. Thus, the task of optimizing timetables requires decision support tools.

A fully automated solution process, however, is not possible, since "winners" and "losers" will inevitably occur while changing timetables and traffic planners have to be able to keep control of the changes made to the network. Even the simple example of two lines that meet at a single station, demonstrated in Figure 1, generates eight transfer possibilities with opposing improvement approaches (a reduction of the waiting time for one transfer directly enforces an extension of the waiting time for an opposing transfer).

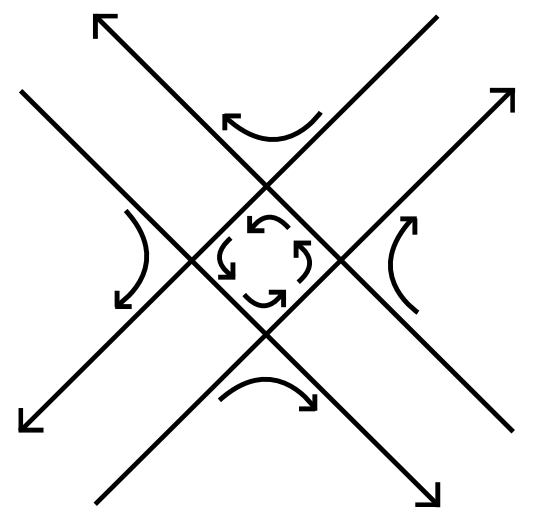

Figure 1: Two lines generate eight different transfer possibilities.

Therefore, we propose an optimization approach that improves the overall situation without losing sight of the deficiencies arising at particular stations. The process interactively incorporates the knowledge and the preferences of the traffic planner and models the problem with respect to the tradeoffs between different transfers.

We demonstrate the model with an example of the public transport network of the city of Kaiserslautern, Germany (ca 100,000 inhabitants and 35 transportation lines of three different companies).

\section{Concept of timetable synchronization}

Our approach is motivated by fundamental considerations of the problem of synchronizing timetables (cf. [1-3]). We believe that the timetable optimization problem cannot be solved solely by an optimization algorithm. An objective 
function that minimizes the average waiting time can work as a rough estimator for the transfer quality, but it is not able to deal with all aspects that make this problem such a hard task to solve.

Existing approaches have, in our opinion, a simplistic model for the decision support of the traffic planner. An a priori specification of all preferences is necessary, but in general it is not possible to set all these preferences in advance. Thus, our approach combines the optimization and the preference acquisition in an interactive process [4].

By introducing the concept of network nodes, we incorporate the fact that not all meetings of two lines generate a real transfer where passengers change between vehicles. These nodes are sets of important stations in the network, where passengers are willing to walk small distances to reach a departing tour. With these network nodes, the number of observed transfers and thus the amount of data is reduced and the focus can be placed on important transfer locations.

\subsection{Main concept}

Timetables are grown structures with established transfer-possibilities where passengers are used to their approved connections. Thus, our goal is not to create a new timetable. Instead, we try to improve the current timetable by applying small changes. Our variables are the given starting times of the lines and tours and we allow small changes to these times to generate better transfer possibilities, especially between different companies. As an example, a shift of +2 minutes to a line means that all buses of this line will start their tours two minutes later.

Several side conditions have to be considered while making these changes. In particular for regional timetables, changes may destroy the vehicle schedules and cause the need for more buses, which is often not marketable due to high acquisition and maintenance costs.

\subsection{Local and global timetable synchronization}

One of the main problems for the traffic planner is the difficulty in acquiring a survey of all transfers in the network manually. But the power of modern computers can assist the planner in getting a quick overview of the global consequences of changes he makes to the network. We call this process of considering the whole network "global timetable synchronization".

On the other hand, it is very important to keep track of the transfers in detail, because some transfers (e.g. important transfers during rush hour traffic) should be preserved by all means. The global optimization has no knowledge about these important transfers, so the traffic planner has to find and mark the ones that should be kept. Furthermore, in some cases the traffic planner wants to generate a new transfer that did not exist before, so he forces the optimization process to change the current timetable such that this new transfer is generated. We call this optimization process that keeps track of detailed information "local timetable synchronization".

The important goal is to find an approach that combines both the global and the local aspects of the problem. As in our opinion it is not possible to create a 
model that directly finds satisfying solutions, we establish an interactive communication process between the traffic planner and the optimization software. The generation of good solutions results from combining mathematical techniques with a decision support concept that helps the traffic planner to determine the direction of the optimization.

\subsection{Partitioning of waiting times}

For creating a good model, we had to develop a way to evaluate transfers (cf. [5]). An easy way is to simply favour shorter waiting times, but for passengers this would not be convenient, since very short waiting times cause the risk to miss a transfer if the arriving tour is behind schedule. Thus, we partitioned the waiting times in five classes.

- Convenience transfers: A waiting time that is not too long and not too short is called "convenient". It secures that small delays do not cause a missed transfer.

- Patience transfers: If a waiting time is too long to be called convenient but still can be seen as a transfer, we call the transfer "patient".

- Risk transfers: A very short waiting time that causes the "risk" to miss the given transfer if the arriving tour is delayed.

- Almost transfers: A small negative waiting time is called an "almost" transfer. The definition of such a transfer is useful, since it describes a very unsatisfactory condition for the passenger. Furthermore, almost transfers can be easily transformed into real transfers by small modifications to the timetable. Thus, they are good starting points for optimization approaches.

- No transfers: We speak of a "no transfer", if no departing tour fits to an arriving tour in a relevant time interval. These not existing transfers are not necessarily bad, since e.g. a connection between a high-frequency line and a low-frequency line causes automatically a lot of "no transfers".

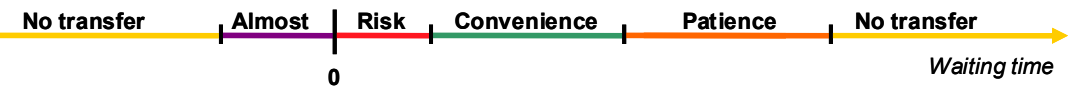

Figure 2: $\quad$ Partitioning of waiting times.

This waiting time classification must be done by the traffic planner in advance to ensure that the optimization results are meaningful. A different waiting time classification is defined for each combination of network node, arriving company, departing company , since e.g. the definition of a risk transfer differs significantly for buses and trains.

For optimization purposes, we introduce a penalty-function for all these partitions. Figure 3 shows an example of such a curve that is used for this purpose. A lower penalty value means a better transfer-possibility for the passenger. 


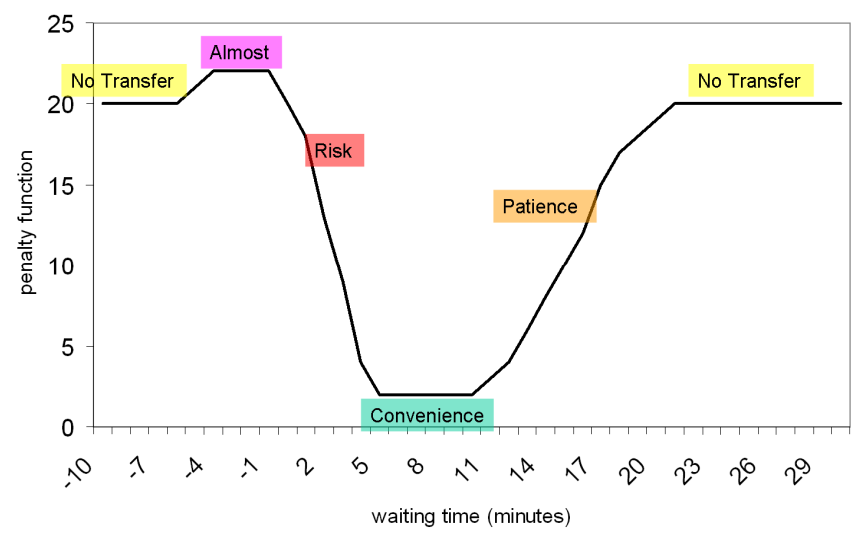

Figure 3: Example of a penalty-function.

\subsection{Transfer ranking versus number of transit passengers}

For getting meaningful results, it is necessary to differ between important and irrelevant transfers. The easiest way to deal with this problem is to take the passenger numbers and calculate weights out of them. These weights could be multiplied with the corresponding penalty-function to achieve higher penalties for more important transfers. The problem of this approach is that the information about the number of passengers is, in most cases, not available on the detail level that is needed.

So our approach is to let the traffic planner rank the transfers on different levels of detail. Thus, each transfer gets three different rankings. These ranks are implemented as an ABC-ranking system (A: important, B: standard, C: less important), from which we calculate weights for each transfer. These weights represent the importance of the transfer for the whole network.

The first ranking level defines how important the place (network node) of the transfer is. The second ranking level compares all combinations of arriving and departing companies within a certain network node. This ranking level expresses whether a transfer is important compared to other transfers at the same network node (e.g. at the main station transfers from train to bus are more important than transfers from bus to bus). The third and most detailed level compares pairs of lines and directions at a network node. It illustrates the importance of special transfers that are more often used by passengers.

\section{Optimization}

The problem of synchronizing timetables can be formulated as a Quadratic SemiAssignment Problem (QSAP) [6], a problem that is known to be hard to solve (NP-hard). A high amount of local minima makes the optimization process very difficult. An impression of these local minima can be seen in Figure 4 that illustrates a small three-dimensional representation of the surface of the problem. 


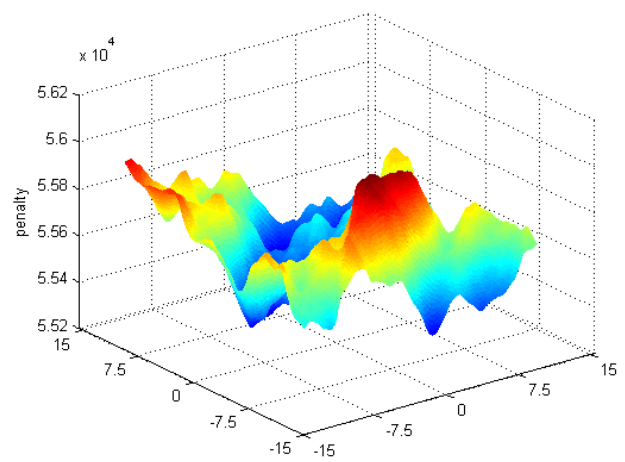

Figure 4: $\quad$ Three-dimensional QSAP surface.

Due to the quadratic terms of the problem, even small instances cannot be solved exactly in reasonable time.

\subsection{There is not a perfect solution}

The given problem, with its thousands of transfers that should all be optimized, has a very high-dimensional structure. Thus, it stands to reason to use multicriteria optimization techniques to solve this problem. In other words, we try to find a good compromise that combines satisfying results for all objective functions.

The mathematical goal is to find solutions that are called Pareto-optimal. These solutions are non-dominated, meaning that they cannot be improved in one objective function without worsening at least one of the others. An important aspect of multi-criteria optimization is that there is not one perfect solution. Instead, the optimization process returns a set of solutions that are all interesting for the user.

The chosen objective functions that guide the optimization process are presented in the following section.

\subsection{Objective functions and constraints}

For the timetable synchronization problem, we identified three main optimization goals. The first one cares about the overall solution quality. Thus, it sums up the quality measure of all transfers in the whole network weighted based on the ABC-rankings.

But this overall view cannot guarantee that the improvement is spread evenly over the network. In the worst case, we have some winner network nodes but for the cost of one big "loser" network node. Since such big losses would cause a lot of frustrated customers, this scenario is not desirable. The second objective function deals with this problem. It minimizes the maximal percental losses at single network nodes. 
The third objective function takes into account that we do not want to create entirely new timetables. Instead, we want to improve the current one by small changes. Thus, the function is closely related to the "mean square error" from the area of stochastics and it measures the amount of changes made to the current timetable.

Constraints represent the wish of the traffic planner to improve the given timetable locally or to preserve good structures that should be kept. During the optimization process, the computer tries to find solutions that fulfil all these constraints and that are still good referring to the objective functions. One main difficulty is the possibility of creating an over-constrained problem, a situation where no solution exists that satisfies all constraints. In such a case, the constraints are considered as soft constraints and a solution that violates the smallest possible number of constraints is desirable.

\subsection{Mathematical tools for the timetable synchronization problem}

A good approach for dealing with combinatorial optimization problems is to model them as a graph-theoretical problem. Such a graph for the timetable synchronization problem, introduced by Voss [6], can be seen in Figure 5.

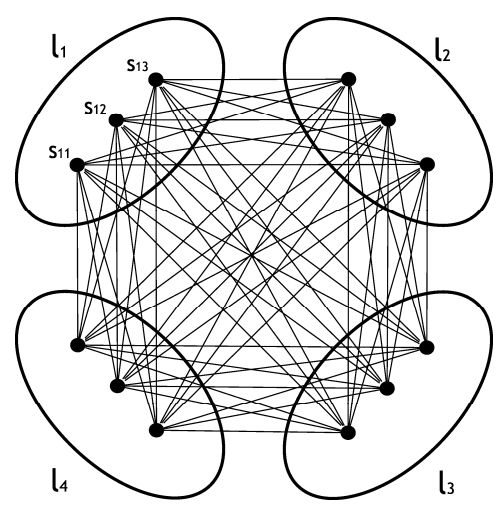

Figure 5: Optimization graph.

The ellipses denote transportation lines $\left\{1_{1}, 1_{2}, \ldots\right\}$ and the vertices represent possible time-shifts $\left\{\mathrm{s}_{11}, \mathrm{~s}_{12}, \ldots\right\}$ for these lines. An edge between two vertices means that the combination of these two shifts is allowed in the given model (if the user forbids certain transfers or has some special wishes for the new timetable, these calibrations are reflected in our model by a removal of edges from the optimization graph). The graph is a multi-partite graph, since there are no edges inside the ellipses, meaning that not two different shifts are allowed for a single line. A solution of the timetable synchronization problem corresponds to a k-clique (a complete graph with $\mathrm{k}$ vertices) in this graph, where $\mathrm{k}$ is the number of lines in the network.

The problem of finding such a clique can be easily formulated as a nonlinear Mixed Integer Program (MIP) that can be solved for one objective function by 
modern solver programs. But since we have a multi-criteria problem, such solvers would need too much time to find the set of non-dominated solutions. Thus, we decided to use a meta-heuristic, an algorithm that is able to generate good solutions (that are not necessarily optimal) in an acceptable time.

From the class of meta-heuristics an evolutionary algorithm, simulated annealing and an ant-colony-optimization algorithm were tested. The results of these tests are presented in [7]. For non-constrained problems, these algorithms behaved similarly. But the more edges were removed from the graph, the better the results of the ant-colony-optimization algorithm became.

\section{Application of the concept}

The given concept was applied to the public transport network of the city of Kaiserslautern (ca 100,000 inhabitants) in the south-west of Germany. There are three main public transport companies in this area, operating the trains (9 lines), the regional buses (12 lines) and the city buses (14 lines). The defined important network nodes were the main station, a smaller train station outside the city and the centralized bus meeting point.

The bus network of Kaiserslautern has a special structure, namely that all city buses periodically meet at certain times at the central station. This concept generates a highly synchronized bus network, but it makes it quite hard to coordinate the buses with the trains.

\subsection{Results}

The presented model was able to reflect the timetable situation of the city of Kaiserslautern in-depth. The optimization procedure automatically detected real life problems that were on the agenda of the traffic planners and it presented new solutions for these problems. In addition, new ideas to improve the connection of the main station and the university could be found. The cooperation with the public transport association of Kaiserslautern showed promising results and a further cooperation is in planning.

Since a full analysis of the results would go beyond of the scope of this paper, we will present two scenarios to give an impression of the outcomes of our approach

\subsection{Scenario 1: status quo analysis}

Timetables are grown structures with established connections and transferpossibilities. Furthermore, passengers are used to their personal connections. Thus, before starting to change the given timetable, the planner has to understand the given situation and to find its strengths and weaknesses. The approach that is presented in this paper gives a simple but powerful possibility for such a status quo analysis. By a classification of the given transfer possibilities in the presented waiting time intervals, one can easily see, whether the situation in a network node or even in the whole system is convenient or not. 
Table 1: $\quad$ Analysis of the status quo situation, Kaiserslautern, Sunday.

\begin{tabular}{|l|c|c|c|c|}
\hline & Almost & Risk & Conv. & Patience \\
\hline Overall & $4.4 \%$ & $2.3 \%$ & $78.9 \%$ & $14.5 \%$ \\
\hline City centre & $2.9 \%$ & $0.3 \%$ & $91.8 \%$ & $5.0 \%$ \\
\hline Main station & $8.5 \%$ & $6.3 \%$ & $46.5 \%$ & $38.7 \%$ \\
\hline Western station & $3.3 \%$ & $16.7 \%$ & $50.0 \%$ & $30.0 \%$ \\
\hline
\end{tabular}

Such an analysis can be seen in Table 1 for the Sunday-timetable of Kaiserslautern.

The analysis shows a highly synchronized situation, especially at the city centre, where more than 90 percent of the arriving buses match conveniently to departing buses of different lines. Improvement potential can be found at the western station, where more than 16 percent of the transfers are risky, but the optimization showed that it is not possible to improve this situation without worsening it elsewhere.

\subsection{Scenario 2: possibilities for improvement}

Without the help of optimization algorithms, it is nearly impossible to find optimal solutions for a new timetable. Nevertheless, the presented concept can be used to generate better solutions with respect to changes to single lines. An example can be given for line 116 that was established some years ago to connect the main station of Kaiserslautern with the university during rush hour times.

Error! Reference source not found. shows a curve that helps the user to evaluate possible changes for a line quickly. The horizontal axis represents the amount of time that is added to the starting times of all buses of line 116 (the vertical line represents a shift of zero minutes); the vertical axis shows the improvement or the worsening of the timetable (a positive value denotes an improvement). The lighter curve stands for newly gained or lost conveniencetransfers and the black curve expresses the overall penalty-function (multiplied by -1 such that higher values represent better solutions).

It can be seen that a shift of -2 minutes to all buses of line 116 will improve the situation.

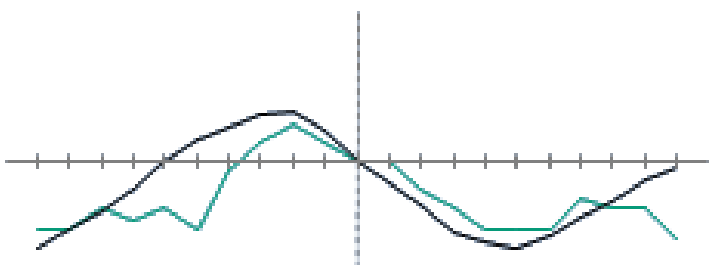

Figure 6: $\quad$ Improvement analysis for line 116. 
This type of analysis can help the traffic planner while constructing new timetables, since it is not just a tool that gives feedback for given changes but it also helps to find appropriate values for new changes. So the planner does not have to use a trial-and-error method, but the curve will give him directly feedback which value is best for the chosen line. Such curves can be easily generated for each line and on each level of detail.

\section{Conclusion}

In this paper we presented a new approach for timetable synchronization in regional public transport systems at the connection points between different operators. This is in line with the overall aim to increase the attractiveness of public transportation in which transferring between vehicles is a major concern for many persons. Convenient transfer options should be offered at as many places and times as possible.

Our approach models the transfer quality in a novel way, distinguishing between convenient, risky or patience-requiring transfers and also modelling explicitly "almost transfers" that are good candidates to be converted into real transfers. This evaluation model is incorporated into an optimization process that uses mathematical optimization algorithms to find small modifications to the operators' timetables that improve the overall transfer quality. This process however is not a black box; the planner can guide it towards desired results by interactively setting constraints addressing local situations.

First results obtained for the public transportation network of the city of Kaiserslautern are very promising. We expect a drastically reduced planning effort for synchronizing timetables of different operators in an urban region by using our approach. Options for optimization that are difficult to detect by manual inspection can be found easily.

We are planning to further analyse our approach. Based on this, our objective is to integrate the use of the method into the routine synchronization process of collaborations of regional public transport operators.

\section{References}

[1] Klemt, W., Stemme. W., Schedule synchronization for public transit networks, J.R. Daduna, A. Wren (eds.), Computer-aided transit scheduling, Proceedings, Lect. Notes Econ. Math. Syst. 308, pp. 327-335, 1988.

[2] Domschke, W., Schedule synchronization for public transit networks, $O R$ Spektrum 11, pp. 17-24, 1989.

[3] Fleurent, C., Lessard, R. Séguin, L., Transit timetable synchronization: evaluation and optimization, $9^{\text {th }}$ International Conference on Computer Aided Scheduling in Public Transport, 2004.

[4] Schroeder, M., Schuele, I., Interaktive mehrkriterielle Optimierung für die regionale Fahrplanabstimmung in Verkehrsverbünden, Straßenverkehrstechnik (6), pp. 332-340, 2008. 
[5] Schroeder, M., Solchenbach, I., Optimization of transfer quality in regional public transit, Berichte des Fraunhofer ITWM, 84, 2006.

[6] Voss, S., Network design formulations in schedule synchronization, $M$. Desrochers et al. (eds.), Computer-aided transit scheduling, Proceedings, Lect. Notes Econ. Math. Syst. 386, pp. 137-152, 1992.

[7] Schuele et al., Multi-criteria optimization for regional timetable synchronization in public transport, Proc. of Int. Conference Operations Research, Augsburg 2008 (to appear). 